\title{
Characteristics of ocular temperature elevations after exposure to quasi- and millimeter waves $(18-40 \mathrm{GHz})$
}

\author{
Masami Kojima • Yukihisa Suzuki • Cheng-Yu Tsai • \\ Kensuke Sasaki • Kanako Wake • Soichi Watanabe • \\ Masao Taki • Yoshitsugu Kamimura • Akimasa Hirata • \\ Kazuyuki Sasaki • Hiroshi Sasaki
}

Received: 20 November 2014 / Accepted: 29 December 2014 /

Published online: 23 January 2015

(C) The Author(s) 2015. This article is published with open access at Springerlink.com

\begin{abstract}
In order to investigate changes in ocular temperature in rabbit eyes exposed to different frequencies (18 to $40 \mathrm{GHz}$ ) of quasi-millimeter waves, and millimeter waves (MMW). Pigmented rabbits were anesthetized with both general and topical anesthesia, and thermometer probes $(0.5 \mathrm{~mm}$ in diameter) were inserted into their cornea (stroma), lens (nucleus) and vitreous (center of vitreous). The eyes were exposed unilaterally to $200 \mathrm{~mW} /$ $\mathrm{cm}^{2}$ by horn antenna for $3 \mathrm{~min}$ at 18,22 and $26.5 \mathrm{GHz}$ using a $\mathrm{K}$ band exposure system or 26.5, 35 and $40 \mathrm{GHz}$ using a Ka band exposure system. Changes in temperature of the cornea, lens and vitreous were measured with a fluoroptic thermometer. Since the ocular temperatures after exposure to $26.5 \mathrm{GHz}$ generated by the $\mathrm{K}$ band and $\mathrm{Ka}$ band systems were similar, we
\end{abstract}

M. Kojima • C.-Y. Tsai $\cdot$ K. Sasaki $\cdot$ H. Sasaki

Division of Vision Research for Environmental Health, Medical Research Institute, Kanazawa Medical

University, Kahoku, Japan

M. Kojima $\cdot$ H. Sasaki

Department of Ophthalmology, Kanazawa Medical University, Kahoku, Japan

M. Kojima $(\bowtie)$

School of Nursing, Kanazawa Medical University, 1-1 Daigaku, Uchinada-machi, Kahoku-gun, Ishikawa 920-0293, Japan

e-mail: m-kojima@kanazawa-med.ac.jp

\section{Y. Suzuki $\cdot$ M. Taki}

Department of Electrical Engineering, Graduate School of Engineering, Tokyo Metropolitan University, Tokyo, Japan

K. Sasaki $\cdot$ K. Wake $\cdot \mathrm{S}$. Watanabe

National Institute of Information and Communications Technology, Tokyo, Japan

Y. Kamimura

Department of Information Science, Graduate School of Engineering, Utsunomiya University, Utsunomiya, Japan

\author{
A. Hirata \\ Graduate School of Engineering, Nagoya Institute of Technology, Nagoya, Japan
}


assumed that experimental data from these 2 exposure systems were comparable. The highest ocular temperature was induced by $40 \mathrm{GHz} \mathrm{MMW}$, followed by $35 \mathrm{GHz}$. The 26.5 and $22 \mathrm{GHz}$ corneal temperatures were almost the same. The lowest temperature was recorded at $18 \mathrm{GHz}$. The elevation in ocular temperature in response to exposure to $200 \mathrm{~mW} / \mathrm{cm}^{2} \mathrm{MMW}$ is dependent on MMW frequency. MMW exposure induced heat is conveyed not only to the cornea but also the crystalline lens.

Key words Quasi-millimeter wave · Millimeter wave · Rabbit eye · Ocular temperature · Frequency-specific difference

\section{Introduction}

Quasi-millimeter waves and millimeter waves (MMWs) are prevalent in high-speed wireless communication, automobile collision prevention systems and high-resolution radar imaging. MMWs have come into widespread use in daily life, leading to increased public concerns about their possible health effects.

The necessity to evaluate the ocular effects of MMW exposure was first mentioned by Gandhi and Riazi in 1986 [1]. Although many studies have assessed the effects of microwave exposure on the eye, few studies have evaluated the specific effects of MMWs [2-6]. Frequency specific thresholds (MMW pulse wave) for ocular damage between 35 and $94 \mathrm{GHz}$ were observed in rhesus monkey eyes, with the mean influence to develop corneal damage being $7.5 \mathrm{~J} / \mathrm{cm}^{2}$ at $35 \mathrm{GHz}$ and $5 \mathrm{~J} / \mathrm{cm}^{2}$ at $94 \mathrm{GHz}$ [4]. In rabbit eyes, $107 \mathrm{GHz}$ effectively produced immediate stromal damage, which was generally gone by the next day, whereas the effects of $35 \mathrm{GHz}$ were persistent and were almost always present the next day [2]. Taken together, these findings suggest that different frequencies have different ocular effects.

The depth of penetration of electromagnetic fields into an irradiated biological body has been shown to decrease as the frequency of the incident field is elevated [7]. Penetration depths of electromagnetic fields also depend on the electrical properties of the biological tissue. The anterior segment of the eye is a complicated structure, being comprised of tear film (3 layers), cornea (5 layers), the anterior chamber (with the aqueous humor circulation dependent on thermal properties), iris (with a 2.5-fold greater blood circulation than the brain [8]), and lens. Thus different wavelengths may have different effects on eye temperature. We have therefore investigated the effects of exposure to MMWs ranging between 18 and $40 \mathrm{GHz}$ on ocular temperature changes in rabbit eyes.

\section{Materials and Methods}

\subsection{Exposure system}

Fig. 1 shows the exposure systems. Continuous waves of 18-26.5 GHz and 26.5-40 GHz were generated by an 83650B (Agilent Technologies, Santa Clara, CA, USA) MMW signal source, driving a $40 \mathrm{~W}$ TWT (18-26.5 GHz K band or 26.5-40 GHz Ka band, ETM Electromatic, Newark, CA, USA) amplifier. The signals were fed to a rectangular horn antenna (QRHKRPS00, Quinstar, Torrance, CA, USA or 12A-26, MI Technologies, Suwanee, GA, USA) to expose rabbit eyes. Both forward and reverse powers were monitored to ensure the desired incident power densities (Fig. 1). 


\section{8-26.5 GHz exposure system ( $\mathrm{K}$ band)}

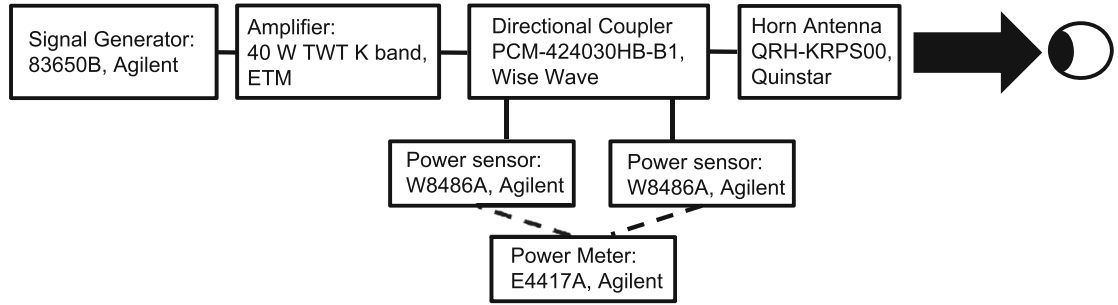

\section{5-40 GHz exposure system (Ka band)}

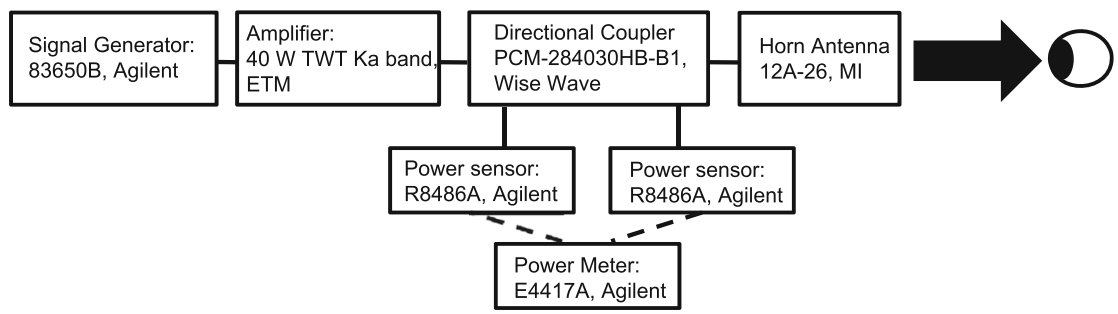

Fig. 1 Block diagram of the MMW exposure system

Rabbit eyes were exposed to MMW of $200 \mathrm{~mW} / \mathrm{cm}^{2}$ for 3 minutes at the distance of $200 \mathrm{~mm}(18-26.5 \mathrm{GHz})$ or $115 \mathrm{~mm}(26.5-40 \mathrm{GHz})$ from the antenna aperture to the corneal apex (Fig. 1). It was confirmed by an exploratory experiment that the corneal surface temperature of rabbit eye reached thermal balance by exposure for 3 minutes. The incident power densities were defined as the average value of $\varphi 13 \mathrm{~mm}$, which nearly corresponds to the surface of the rabbit cornea.

The center of the corneal surface was positioned on the line of the maximum radiation of the antenna. The exposure point $(200 \mathrm{~mm}(18-26.5 \mathrm{GHz})$ or $115 \mathrm{~mm}(26.5-40 \mathrm{GHz})$ distance from the antenna surface to corneal apex) was set by red and green laser pointers on a target (Fig. 2A). After removal of the target, the apex of rabbit's cornea was positioned at the exposure point (Fig. 2B).

Although our exposure system was not used for far-field conditions, the distributions of the incident power density were nearly uniform. The variation of the incident power densities at the area of $\varphi 13 \mathrm{~mm}$ (similar size of cornea surface) was lower than $1 \mathrm{~dB}$ for all frequencies used in this study.

\subsection{Experimental animals}

All animal experiments were conducted in accordance with the animal study guidelines established by Kanazawa Medical University (Kahoku, Japan) and the ARVO Statement for the Use of Animals in Ophthalmic and Vision Research [9].

Specific pathogen-free (SPF) pigmented male Dutch-belted rabbits of age 11-13 weeks and body mass 1.9-2.4 kg were purchased from Sankyo Lab Service (Toyama, Japan). They were kept in SPF cages with unrestricted access to sterilized food and ultraviolet-irradiated water. The eyes of each rabbit were examined with an SL-130 slit-lamp microscope (Zeiss, Tokyo, Japan) to ensure absence of abnormalities in the anterior segment and the image was recorded as the pre-exposure condition. 

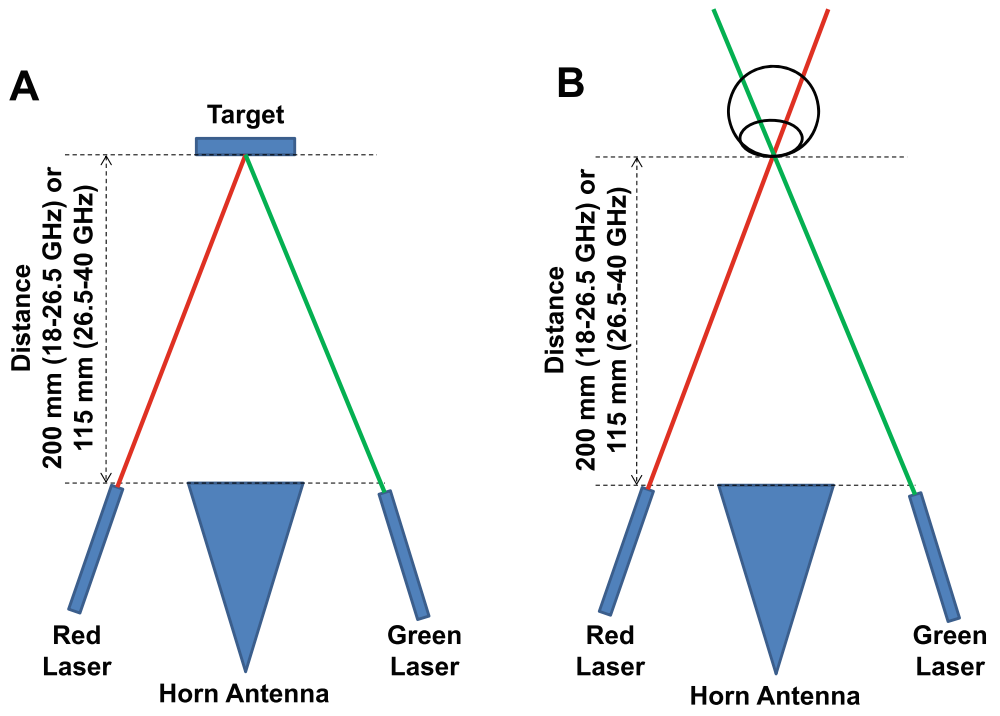

Fig. 2 Eye positioning system

Each rabbit was immobilized using a lucite animal holder, specially constructed for this study, and injected intramuscularly with a solution containing $2 \mathrm{mg} / \mathrm{kg}$ of xylazine and $20 \mathrm{mg} /$ $\mathrm{kg}$ of ketamine to induce general anesthesia. Immediately before MMW exposure, a $2 \%$ lidocaine hydrochloride topical anesthetic (Xylocaine 2\%; AstraZeneca, Osaka, Japan) was administered to each eye. The upper and lower eyelids were retracted with tapes to keep the eyes open during MMW exposure. A cooling pad (Hiepita for Kids, Lion, Tokyo, Japan) was used to avoid heating the tissue surrounding the eyes.

Since anesthesia suppressed blinking, we administered $0.1 \%$ sodium hyaluronate (Hyaline Mini ophthalmic solution 0.1\%; Santen Pharmaceutical, Osaka, Japan) eye drops, pre-warmed to $35-37{ }^{\circ} \mathrm{C}$, as necessary to each eye to prevent corneal desiccation and the resulting damage to corneal epithelial cells; by pre-warming the eye drops, we minimized their effects on corneal temperature changes.

\subsection{Ocular temperature measurements}

Temperatures during MMW exposure were measured with a Fluoroptic Thermometer (Luxtron 790, Luxtron, Santa Clara, CA, USA), which was set up as described [10]. After both general and topical anesthesia, thermometer probes $(0.5 \mathrm{~mm}$ in diameter $)$ were inserted into the corneal stromal layer, lens nucleus and the center of vitreous, with the tip of each probe positioned at the center of the pupillary area. The thermometer probes were inserted under slit-lamp microscopy and their positions were checked by slit-lamp photography. After the corneal basal temperature stabilized, the topical anesthetic (warmed up to around 35-37 ${ }^{\circ} \mathrm{C}$ ) was instilled into the conjunctival sac. The resulting corneal temperature drop down around 1 or $2{ }^{\circ} \mathrm{C}$. Once the corneal temperature stabilized again, MMW exposure was started. The rabbit eyes were unilaterally and sequentially exposed to $200 \mathrm{~mW} / \mathrm{cm}^{2}$ at $18 \mathrm{GHz}, 22 \mathrm{GHz}$ and $26.5 \mathrm{GHz}$ or at $26.5 \mathrm{GHz}, 35 \mathrm{GHz}$ and $40 \mathrm{GHz}$, in ascending or descending order.

To improve the accuracy of measurement, the average of 3-5 ocular temperature measurements from one rabbit was considered as one measurement. To consider an animal's individual 
specificity, 6-8 rabbits' mean values were compared with each frequency. Room temperature and humidity during MMW exposure were maintained at $20-25{ }^{\circ} \mathrm{C}$ and $33-43 \%$ respectively, through air-conditioning.

\subsection{Visualization of heat transportation in the anterior chamber}

Microencapsulated thermochromic liquid crystals (MTLC, Japan Capsular Products, Tokyo, Japan) are 20-30 micrometer particles, the color of which changes as the surrounding temperature changes [11-13]. The coloration range of MTLC is 35 to $45{ }^{\circ} \mathrm{C}$. They turn red at $35^{\circ} \mathrm{C}$, then yellow to green at just below to just above $39^{\circ} \mathrm{C}$, and blue at $42{ }^{\circ} \mathrm{C}$. Using a 30 gauge injection needle and syringe, $0.2 \%$ MTLC was injected into the anterior chamber of each rabbit eye. The rabbit eyes were then unilaterally exposed to $200 \mathrm{~mW} / \mathrm{cm}^{2}$ at $18 \mathrm{GHz}$ or $40 \mathrm{GHz}$. A video camera was used to record aqueous convection and change in MTLC color in the anterior chamber.

\subsection{Data analysis}

Differences between outcomes of the K band (18-26.5 GHz) and Ka band (26.5-40 GHz) exposure systems were analyzed using Student's two-tailed t-test, with a $\mathrm{P}$ value $<0.05$ considered statistically significant. The relation between ocular temperature elevation and frequency was analyzed using general linear mixed model (JMP ${ }^{\circledR} 7$, SAS Institute, Tokyo, Japan).

\section{Results}

Fig. 3 shows the average ocular temperature changes after exposure of eyes to $200 \mathrm{~mW} / \mathrm{cm}^{2}$ at $26.5 \mathrm{GHz}$ using the two exposure systems. There were no statistical differences in the temperatures of the cornea, lens and vitreous using these two systems at the same wavelength.

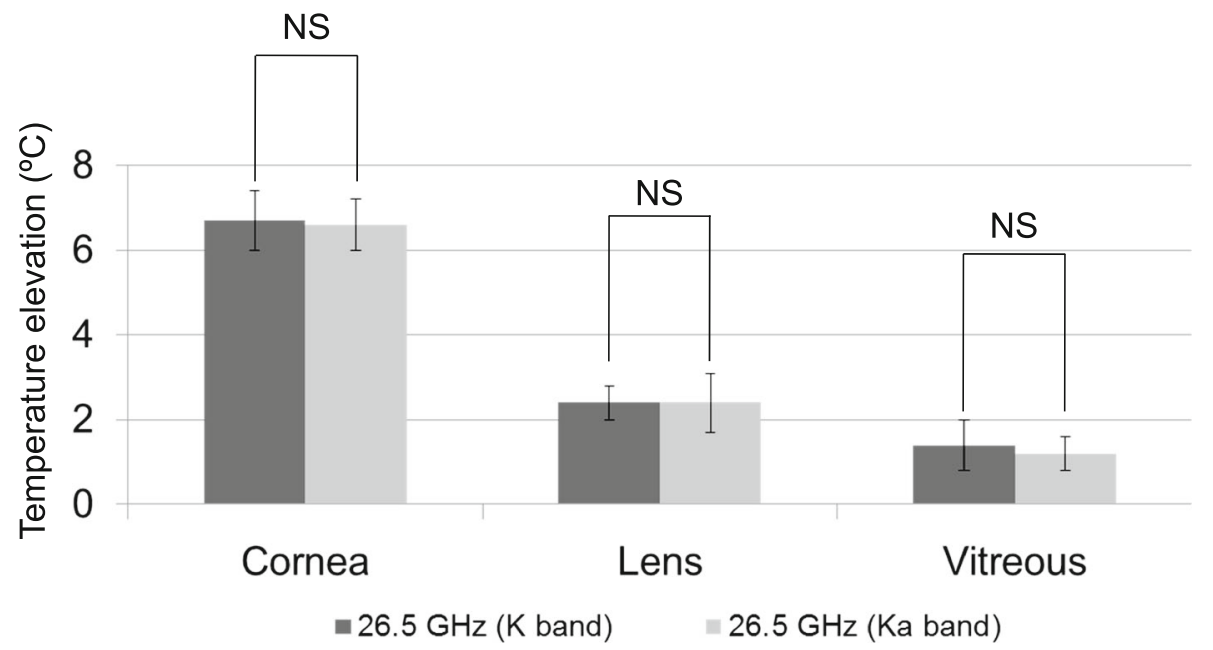

Fig. 3 Ocular temperature changes induced by the K and Ka band exposure systems at $26.5 \mathrm{GHz}$. NS indicates not significant difference 
Since these ocular temperatures under exposure $26.5 \mathrm{GHz}$ corresponded, we regarded further experimental data obtained from these 2 exposure systems as comparable.

Fig. 4 shows temperature elevation by frequency $(18,22,35,40 \mathrm{GHz}$, averages of $6-8$ rabbits: $26.5 \mathrm{GHz}$, average of 16 rabbits) during exposure to $200 \mathrm{~mW} / \mathrm{cm}^{2}$ at 18 or $40 \mathrm{GHz}$. Elevation of the corneal temperature was the highest, followed by elevation of the lens temperature and then the vitreous temperature. In general, higher frequencies showed higher ocular temperature elevations (Trend: cornea $(p<0.001)$, lens $(p<0.003)$, vitreous (not significant)). Although all eyes were exposed to the same incident power density of MMWs, those exposed to $40 \mathrm{GHz}$ had a 1.6-fold higher corneal temperature than those exposed to $18 \mathrm{GHz}$.

Fig. 5 shows differences in time-course change of temperature in anterior chamber by 18 or $40 \mathrm{GHz}$. The area of the upper anterior chamber located between the cornea and iris showed an MTLC red-yellow color tone change 5 seconds after the start of $18 \mathrm{GHz} 200 \mathrm{~mW} / \mathrm{cm}^{2}$ exposure (Fig. 5A). In contrast, the upper part of the anterior chamber showed an MTLC red-yellow-green color tone change, and the lower part showed a green color tone change, 5 seconds after the start of $40 \mathrm{GHz} 200 \mathrm{~mW} / \mathrm{cm}^{2}$ exposure (Fig. 5D). MTLC color tone change by $18 \mathrm{GHz} 200 \mathrm{~mW} / \mathrm{cm}^{2} 10$ second exposure was seen only in the upper part of the anterior chamber, but MTLC color tone change by $40 \mathrm{GHz} 200 \mathrm{~mW} / \mathrm{cm}^{2} 10$ second exposure was seen in both upper and lower parts of the anterior chamber (Fig. 5B and 5E).

$18 \mathrm{GHz} 200 \mathrm{~mW} / \mathrm{cm}^{2} 30$ seconds exposure caused an MTLC blue (over $42{ }^{\circ} \mathrm{C}$ ) color tone change in the upper part of the anterior chamber and an MTLC green (around $39{ }^{\circ} \mathrm{C}$ ) color tone change in the center and lower parts (Fig. 5C). In contrast, an MTLC blue (over $42{ }^{\circ} \mathrm{C}$ ) color tone change was seen in the upper and lower of the anterior chamber exposed to $40 \mathrm{GHz}$ $\left(200 \mathrm{~mW} / \mathrm{cm}^{2}\right) 30$ seconds after the start of exposure (Fig. 5F). MTLC color analysis revealed that although both eyes were exposed to the same incident power density of MMWs, $40 \mathrm{GHz}$ exposure induces a higher temperature than does $18 \mathrm{GHz}$, in agreement with findings that $40 \mathrm{GHz}$ had a 1.6-fold higher corneal temperature effect than that of $18 \mathrm{GHz}$ in the cornea and lens temperature (Fig. 4).

Cornea

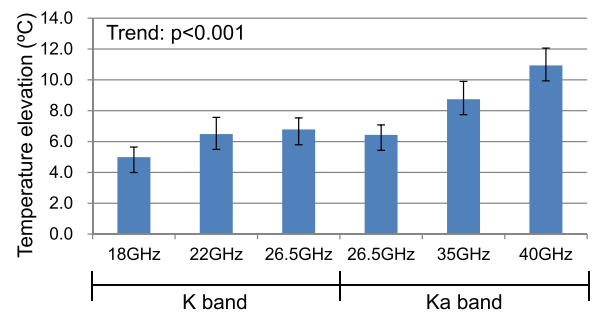

\section{Lens}

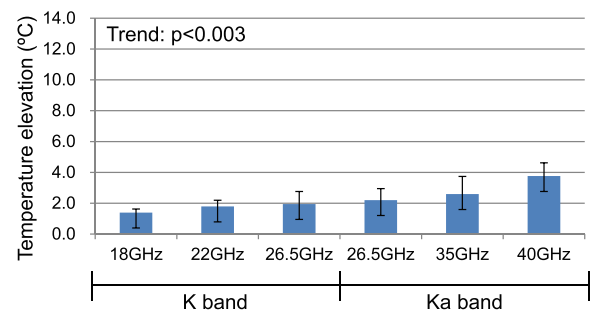

Vitreous

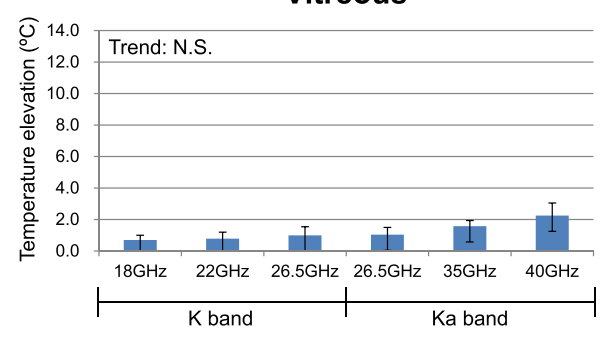

Fig. 4 Temperature elevation during exposure to $200 \mathrm{~mW} / \mathrm{cm}^{2}$ at 18 and $40 \mathrm{GHz}(\mathrm{K}$ and $\mathrm{Ka}$ band exposure systems) 


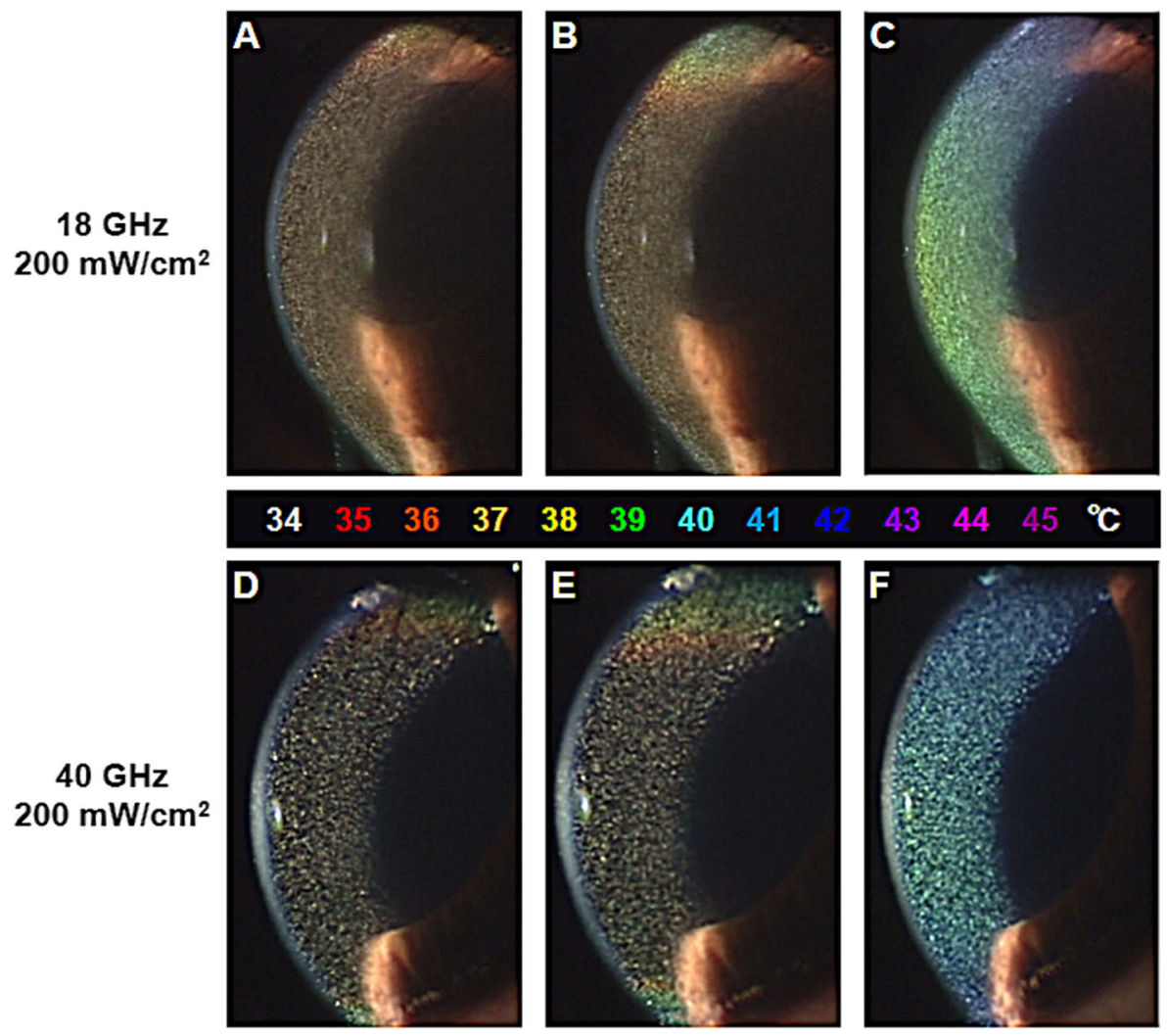

Fig. 5 Temperatures of anterior chamber measurement by MTLC during MMW exposure. $18 \mathrm{GHz} 200 \mathrm{~mW} /$ $\mathrm{cm}^{2}$ : A: 5 seconds after the start of exposure, B: 10 seconds after the start of exposure, $\mathbf{C}: 30$ seconds after the start of exposure. $40 \mathrm{GHz} 200 \mathrm{~mW} / \mathrm{cm}^{2}$ : D: 5 seconds after the start of exposure, E: 10 seconds after the start of exposure, F: 30 seconds after the start of exposure

Nevertheless, additional studies are needed to clarify the relationship between ocular heat transport and aqueous humor convection.

\section{Discussion}

The safety guidelines of the International Commission on Non Ionizing Radiation Protection [14] and the International Committee on Electromagnetic Safety (ICES) of the Institute of Electrical and Electromagnetic Engineers [15] are similar for exposures up to $300 \mathrm{GHz}$. Guidelines for MMWs are mainly based on the threshold of thermal sensations, but these guidelines are unclear when considering thermal effects on the eye.

Thermal ocular effects have been reported to differ under exposure to $35 \mathrm{GHz}$ or $107 \mathrm{GHz}$ MMWs [2]. Although both induced similar corneal damage immediately after exposure, the effects of exposure to $35 \mathrm{GHz}$ continued longer than those of exposure to $107 \mathrm{GHz}$, a difference that may be due to wavelength specificity or relative biological effectiveness. In contrast, a comparison of the threshold of tissue damage between 35 and $94 \mathrm{GHz}$ found that the higher frequency had a lower tissue damage threshold [4]. 
Depth of penetration of electromagnetic fields into an irradiated object decreases as the frequency of the incident field elevations [7]. Therefore, people believe that in the eye, higher frequencies penetrate only the surface of the cornea. Moreover, people believe that energy absorbed at the corneal surface (i.e., heat) can easily radiate in air. Indeed, ICNIRP guidelines indicate that the portion of the eye which absorbs very high frequencies (10 to $300 \mathrm{GHz})$ is the surface of the eye [14]. Hagan and Carpenter [16] compared cataractogenic potential between $2.45 \mathrm{GHz}$ and $10 \mathrm{GHz}$ and found that $2.45 \mathrm{GHz}$ has greater cataractogenic potential than 10 GHz. The reason is consistent with knowledge of peak energy absorption, that the higher frequency $(10 \mathrm{GHz})$ is absorbed at the outer surface of the eye and not in or near the lens.

To confirm the frequency specificity of quasi-MMW and MMW bands, we compared ocular temperatures during exposure to $200 \mathrm{~mW} / \mathrm{cm}^{2}$ at $18,22,26.5,35$ and $40 \mathrm{GHz}$. In addition, we designed a method to measure transport of heat in the eye induced by quasi MMW or MMW exposure.

The experimentally measured rabbit corneal temperature showed a temperature elevation during exposure. The experimental temperature measurements showed that a rise in frequency from 18 to $40 \mathrm{GHz}$ created a higher temperature (Fig. 4). This is consistent with previous data about human skin warmth sensations [17].

The ocular temperature measurement during exposure from 18 to $40 \mathrm{GHz}$ showed not only corneal temperature elevation, but also lens and vitreous humor temperature elevation during exposure (Fig. 4).

Temperature measurements in the anterior chamber by MTLC (although these measurements were semiquantitative) during MMW exposure showed that $40 \mathrm{GHz}$ exposure induces a higher temperature than $18 \mathrm{GHz}$ exposure in the anterior chamber. The color tone of MTLC on the surface of the crystalline lens 30 seconds after exposure start was almost the same as that directly under the cornea (Fig. 5C and 5F). The result of this MTLC experiment shows that heat by MMW exposure reached the crystalline lens. Examination by MTLC in non-exposed rabbit eye did not reveal such temperature differences indicating changes were due to MMW exposure and not intrinsic to rabbit eye. (Data not shown).

It is not yet clear whether the mechanism of intraocular heat transportation is conduction, transportation by aqueous humor convection, or both. It is clear, however, that quasi-MMW and MMW exposure induced heat is conveyed not only to the cornea but also the crystalline lens.

This phenomenon may suggest that aqueous humor convection has an important role in the heat transfer from the cornea to the lens or vitreous humor.

Karampatzakis and Samaras reported that aqueous humor flow in the anterior chamber had an important role in heat transfer using a 3D numerical model [18]. We need further study to clarify the relation between ocular heat conduction and aqueous humor convection.

We found the elevation in corneal, lens and vitreous temperature was associated with frequency. Our temperature measurement probe size is $0.5 \mathrm{~mm}$ and the wavelengths of MMW-band are several millimeter in the ocular tissue. The effects in temperature measurement due to field distortion caused by temperature probe insert might not be negligible. The results obtained in this study reflect the tendency for actual temperature elevations, although the measured temperatures may include some errors.

Further experiments are necessary to assess thermal transport in the eyeball because there are tear film layers at the front of the cornea and aqueous humor convection in the anterior chamber. The corneal surface is covered by a thin tear film, which protects the cornea from direct exposure to air, which can damage the corneal epithelium. The thermal effects of MMW exposure are likely more severe in the eyes of individuals with dry eye, whose tear film is much thinner and easier to penetrate, than in individuals with normal eyes. Since the 
prevalence of dry eye is very high and elevations with age, differences between dry and normal eyes should be considered in the assessment of the effects of MMWs in future work.

\section{Conclusion}

We have shown here that the elevation in ocular temperature in response to exposure to 200 $\mathrm{mW} / \mathrm{cm}^{2} \mathrm{MMW}$ is dependent on MMW frequency. Exposure to $40 \mathrm{GHz}$ MMWs resulted in a 1.6-fold higher corneal temperature than exposure to $18 \mathrm{GHz}$ MMWs. MMW exposure induced heat is conveyed not only to the cornea but also the crystalline lens. Further experiments are needed to elucidate the mechanism(s) underlying frequency-specific differences in ocular temperature elevations.

Acknowledgements This work was supported by the Committee to Promote Research on the Potential Biological Effects of Electromagnetic Fields, Ministry of Internal Affairs and Communications, Japan.

The authors thank to Dr. Taiji Sakai and Ms. Yoko Yamashiro for their technical assistance and Mr. David Price for the professional medical English editing.

Open Access This article is distributed under the terms of the Creative Commons Attribution License which permits any use, distribution, and reproduction in any medium, provided the original author(s) and the source are credited.

\section{References}

1. O. P. Gandhi, A. Riazi, Absorption of millimeter waves by human beings and its biological implications, Microwave Theory and Techniques, IEEE Transactions, 34, 228-235 (1986).

2. S. W. Rosenthal, L. Birenbaum, I. T. Kaplan, W. Metlay, W. Z. Snyder, M. M. Zaret, Effects of 35 and $107 \mathrm{GHz}$ CW microwaves on the rabbit eye. In: C. C. Johnson, M. L. Shore (eds), Biological effects of electromagnetic waves selected papers of the USNC/URSI annual meeting. Boulder. Colorado. October 1975 Rockville. Maryland. US Department of Health, Education, and Welfare: HEW Publication (FDA) 778010, 110-128 (1976).

3. H. A. Kues, S. A. D’Anna, R. Osiander, W. R. Green, J. C. Monahan, Absence of ocular effects after either single or repeated exposure to $10 \mathrm{~mW} / \mathrm{cm}^{2}$ from a $60 \mathrm{GHz} \mathrm{CW}$ source, Bioelectromagnetics, 20, 463-473 (1999).

4. S. Chalfin, J. A. D’Andrea, P. D. Comeau, M. E. Belt, D. J. Hatcher, Millimeter wave absorption in the nonhuman primate eye at $35 \mathrm{GHz}$ and $94 \mathrm{GHz}$, Health Phys., 83, 83-90 (2002).

5. M. Kojima, M. Hanazawa, Y. Yamashiro, H. Sasaki, S. Watanabe, M. Taki, Y. Suzuki, A. Hirata, Y. Kamimura, K. Sasaki, Acute ocular injuries caused by $60-\mathrm{GHz}$ millimeter-wave exposure, Health Phys., 97, 212-218 (2009).

6. K. R. Foster, J. A. D'Andrea, S. Chalfin, D. J. Hatcher, Thermal modeling of millimeter wave damage to the primate cornea at $35 \mathrm{GHz}$ and $94 \mathrm{GHz}$, Health Phys., 84, 764-769 (2003).

7. C. Durney, H. Massoudi, M. Iskander, Radiofrequency radiation dosimetry handbook, 4th edn. (US Air Force School of Aerospace Medicine, Technical Report USAFSAM 85-73, Texas, Brooks Air Force Base, 1986)

8. A. Alm, Ocular circulation, in Adler's Physiology of the Eye, ed. By W. M. Hart, Jr. (C.V. Mosby, St. Louis, USA, 1992), p. 198-227

9. ARVO Statement for the Use of Animals in Ophthalmic and Vision Research http://www.arvo.org/Journals and_Publications/Toolkit_for_Biomedical_Researchers_Using_Laboratory_Animals/. Accessed 17 November, 2014.

10. M. Kojima, I. Hata, K. Wake, S. Watanabe, Y. Yamanaka, Y. Kamimura, M. Taki, K. Sasaki, Influence of anesthesia on ocular effects and temperature in rabbit eyes exposed to microwaves, Bioelectromagnetics, 25, 228-233 (2004).

11. J. L. Fergason, Liquid crystals in nondestructive testing, Appl. Opt., 7, 1729-1737 (1968). 
12. D. Dabiri, M. Gharib, Digital particle image thermometry: The method and implementation, Exp. Fluids., 11, 77-86 (1991).

13. Y. Suzuki, M. Baba, M. Taki, K. Fukunaga, S. Watanabe, Imaging the 3D temperature distributions caused by exposure of dieelectric phantoms to high-frequency electmagnetic fields, IEEE., 13, 744-750 (2006).

14. International Commission on Non-Ionizing Radiation Protection. Guidelines for limiting exposure to time varying electric, magnetic and electromagnetic fields (up to $300 \mathrm{GHz}$ ), Health Phys., 74, 494-522 (1998).

15. IEEE International Committee on Electromagnetic Safety. IEEE Standard for Safety Levels with Respect to Human Exposure to Radio Frequency Electromagnetic Fields, $3 \mathrm{kHz}$ to $300 \mathrm{GHz}$. IEEE Std C951 (2005).

16. G. J. Hagan, R. L. Carpenter, Relative cataractogenic potencies of two microwave frequencies (2.45 and 10 $\mathrm{GHz}$ ). in Biological effects of electromagnetic waves. eds. By C. C. Johnson, M. L. Shore (Rockville, MD: HEW Publication (FDA), 77-8010, 1976), p. 143-155

17. P. J. Riu, K. R. Foster, D. W. Blick, E. R. Adair, A thermal model for human thresholds of microwaveevoked warmth sensations, Bioelectromagnetics, 18, 578-583 (1997).

18. A. Karampatzakis, T. Samaras, Numerical model of heat transfer in the human eye with consideration of fluid dynamics of the aqueous humour, Phys Med Biol., 55, 5653-5665 (2010). 